\title{
Embodiment in Affective Space: Social Influences on Spatial Perception
}

\author{
Simone Schnall ${ }^{1}$
}

\begin{abstract}
Unlike social judgments, perceptual judgments are anchored in concrete reality and should not depend on social context. However, recent research suggests that perceptions of physical space can depend on social and emotional considerations. In contrast to theoretical approaches that view visual perception as a low-level process that is entirely independent of situational constraints, many studies support the notion that visual perception takes place in an "embodied" fashion, because people perceive the physical world around them as a function of how they would act in that world. This chapter reviews the influence of social and emotional factors in research involving maps and other conceptual representations of space, and in research involving the perception of distances and inclines. The reviewed findings provide a glimpse of how social factors influence basic cognitive processes previously assumed to be insulated from such influences.
\end{abstract}

\section{Introduction}

We speak of A as a 'close' friend, B as a 'distant' sort of person. We keep C at 'arm's length,' consider E 'aloof' or 'withdrawn,' $\mathrm{F}$ as 'pushy' and with some scientific rigor characterize interpersonal relationships in terms of 'social distance.' (Little, 1965, p. 238, emphasis added).

Metaphors describing social relationships in terms of distance are plentiful (Lakoff \& Johnson, 1980; 1999). These metaphors are not arbitrary: People use space in specific ways as a function of social relationships, as shown by Proxemics, the study of personal space (Argyle \& Dean, 1965; Hall, 1968; Hayduk, 1983). For example, people maintain less distance when they feel "close" to somebody (Patterson, 1977; Willis, 1966), whereas they main-

1 Preparation of this paper was supported by NSF grant BCS 0518835 and ESRC First Grant RES-061-25-0119. Thanks to the Department of Psychology at Harvard University for accommodating the author as a Visiting Scholar during the Spring of 2009 when this paper was prepared. 
tain more distance when the other person is disliked, or carries a physical stigma (Kleck, 1968). Further, people maintain an area of personal space around them that is usually not violated by others (Hall, 1968). Thus, social factors often influence how people interact in physical space. Further, recent research has explored the extent to which perceived distance expands or contracts depending on how "close" or "distant" a social relationship is. This chapter will illustrate how social distance is related to perceived distance, and more generally, how social and affective factors influence perception of physical space.

In line with the claim that cognitive processes are embodied and actiondriven, spatial perception appears to be constrained by a person's potential to carry out specific actions in a given environment. Recent work by Proffitt and colleagues supports the idea that functional aspects play a critical role in perception. For example, the perception of slants of hills and of distances is influenced by factors such as whether the perceiver is wearing a heavy backpack (Proffitt, Stefanucci, Banton, \& Epstein, 2003), is young or old (Bhalla \& Proffitt, 1999), is fatigued (Proffitt, Bhalla, Gossweiler, \& Midgett, 1995), or has action goals in mind (Witt, Proffitt, \& Epstein, 2004). Such factors influence perception because they are relevant to action. For instance, a distance is harder to traverse for an elderly, or a fatigued person, and thus, appears as farther. These studies suggest that visual perception combines aspects of the perceiver with the environment to be perceived. Of primary concern is what Proffitt (2006) calls the economy of action. In order to plan and to manage one's physical and mental resources to cope with the world, humans, and indeed all organisms, scale the world in terms of the actions that are afforded by their bodies. According to this view, visual perception provides a mechanism to indicate whether exerting a certain amount of energy is worthwhile, given the potential energetic costs and current resources available: Plans for action are related to their energetic costs, and people are informed about these contingencies through their visual perception, because it reflects, given the current state of the body, how difficult and costly it would be to perform a given action in the environment.

With respect to the two important geometric and psychological parameters that have been studied - slant and extent - it can be argued that social resources may inform perception of the world no less crucially than does the availability of our physical energy. Just as steep hills and great distances require that physical energy be available, they may also require emotional and social resources. The larger idea is that our perception of the physical world, as well as of psychological situations, is constrained by emotional and social factors. All these influences constrain perception, because they are concerned with goals, plans, and resource decisions, or 
more generally, the anticipation of action. It is because of this motivation to take action that functional demands of a situation make a person literally look at the world differently.

To some extent perceptual estimates of the physical world, namely estimates of distances and of hill slant, might be similar to the kinds of judgments studied by social psychologists, such as ratings of life satisfaction, probability, risk, and so on. The latter kinds of judgments are characterized by two aspects: First, they are "subjective." For example, the same circumstances can result in high ratings of life satisfaction for one person, but low ratings of life satisfaction for another person. Second, judgments depend on various properties of the context in which the judgments are made. Such contextual factors include the framing of the question (Tversky \& Kahneman, 1981), the mood of the person being questioned (e.g., Schwarz \& Clore, 1983; Schnall, Haidt, Clore \& Jordan, 2008), currently accessible cognitive concepts (e.g., Schnall, Benton \& Harvey, 2008), and so on. Perceptual estimates may share these two aspects of judgments, and are sensitive to the demands of a given context. However, perceptual estimates differ in one important way from other judgments: They can be more or less accurate. In contrast to judgments of liking, life satisfaction, moral integrity, etc., there is a correct answer when it comes to perceptions of the real world: A $12 \mathrm{~cm}$ long line on a piece of paper, such as a map, simply is $12 \mathrm{~cm}$ long.

In this chapter research concerning small-scale distance estimates on maps and other visual media will be reviewed first, and how recent work in this domain has started to incorporate social and emotional considerations of the perceiver. Following this, recent developments in cognitive science related to embodied cognition will be discussed. Then research on embodied perception, involving perceptual estimates in the physical environment such as perceptions of hill slant and of distance will be reviewed. The next section will discuss how social and emotional factors inform spatial perception, and parallels between physiological and social resources will be explored. Finally, similarities of subjective and objective judgments within cognitive and social psychology will be considered.

\section{Physical and psychological maps}

In order to move around in the world individuals need to have an internal representation of the environment to consult for navigation. However, cognitive distance, defined as the spatial component of an individual's representations of the environment, differs from actual, or physical distance (Golledge, 1987), and can be changed by structural properties of space 
(Tversky, 2000). For example, people appear to divide the environment into meaningful categories ("chunks") which subsequently influence spatial judgments, leading to distortions when estimating distances on maps and other visual media. For example, Thorndyke (1981) had participants study maps with city names, and then estimate the distance between pairs of cities. The greater the number of intervening cities between two target cities, the greater was the estimated distance between those two cities. In addition, distances between stimuli that are considered part of a perceptual Gestalt are underestimated compared to stimuli that are outside of that Gestalt (Coren \& Girgus, 1980). Thus, when crossing over a boundary, whether physical or conceptual, subjective distance estimates increase.

Additional research has investigated whether subjective psychological feelings associated with crossed borders might also change distance estimates. Indeed, attitudes related to in-groups and out-groups have been shown to influence distance estimates in recent studies (Kerkman, Stea, Norris, \& Rice, 2004; Burris \& Branscombe, 2005). For instance, Burris and Branscombe (2005) asked university students from Memphis, Tennessee, and Lawrence, Kansas, to estimate distances between cities on a map of the United States. Participants overestimated the distances between a U.S. location and a foreign location relative to a visually equidistant U.S. location. Of primary interest was that this overestimation took place only when the distance estimates involved crossing the U.S. border to a foreign country such as Canada or Mexico, but not when it involved crossing borders between two foreign locations. Burris and Branscombe (2005) interpreted their data as suggesting the effect a "psychological boundary" between relevant space to oneself and one's ingroup, namely one's own country, and nonself-relevant space, namely other countries. Similarly, Carbon and Leder (2005) showed self involvement as a potential factor for a bias in distance estimation across borders of countries. They found that distances between city pairs that involved crossing the former "Iron Curtain", with one city in East Germany, and the other city in West Germany, were systematically overestimated compared to distances of cities located within the same parts of Germany. Interestingly, this overestimation of distances between regions was especially pronounced for participants who exhibited a negative attitude toward the reunification of Germany.

Bugmann and Schnall (2009) recently showed that experimentally induced negative emotions can also change estimates of distances between cities on a map. Because disgust is considered to be evolutionarily significant in protecting one's physical boundaries (Haidt, Rozin, McCauley \& Imada, 1997), Bugmann and Schnall (2009) manipulated disgust by having participants fill out the Disgust Sensitivity Scale (Haidt, McCauley \& Rozin, 1994), which involves considering a variety of potentially disgust- 
ing situations. After being induced to feel disgust participants systematically gave higher distance estimates between city pairs than participants who were in a neutral emotional state. This finding suggests that incidental feeling of disgust can establish a psychological boundary involving a desire to remove oneself from an unpleasant source of affect, and as a consequence influences cognitive distance even when the disgust is not relevant to the current situation.

Several recent studies demonstrated that metacognitive factors, such as processing fluency and primed perceptual anchors can change distance estimates. Alter and Oppenheimer (2008) asked participants to consider distances between cities as they were waiting at a train station in New Jersey, and estimated distances to a variety of US cities from their current location. Some of the participants received the questionnaire printed in a font that was easy to read, thus involving a metacognitive state of perceptual fluency (e.g., Winkielman, Schwarz, Fazendeiro, \& Reber, 2003), whereas other participants received font that was difficult to read, thus involving perceptual disfluency. When reading the questionnaire was difficult and required effort, participants gave higher distance estimates between the cities than when reading the questionnaire did not require effort. Presumably, the disfluency experienced when reading the item was interpreted to indicate that distances between the cities must be far.

LeBoef and Shafir (2006) asked participants to draw a 3.5 inches long line on a sheet of paper. Half of the participants started with a very short line and had to complete it to make it the required length, whereas the other half of them started with a long line and had to shorten it. Those extending the previously given short line drew significantly shorter lines than those shortening the given long line, suggesting that whatever anchor a participant started with determined how long they perceived 3.5 inches to be.

All these findings suggest that rather than being objectively determined by a low-level modular process (Fodor, 1983), visual processes such as estimating small-scale distances on maps are constrained by various contextual factors, which can include mood, processing fluency, and cognitive anchors. The work reviewed above concerned spatial estimates that involved representations of space in the form of maps, or lines on paper. Other research has investigated contextual influences on the perception of physical space to which observers are directly exposed; for example, participants might stand at the bottom of a hill and estimate its slant, or stand in a grassy field and estimate distance to specific targets. Such direct perceptual estimates of physical space are also constrained by contextual factors, which is in line with one of the central tenet of theories of embodied cognition that have become prominent in recent years, as will be reviewed next. 


\section{Embodied cognition}

Following researchers in other areas of cognitive science (e.g., Barsalou, 1999; Clark, 1997; Glenberg, 1997; Lakoff \& Johnson, 1980; 1999; Varela, Thompson \& Rosch, 1991), social psychologists have started to emphasize the benefits of an embodied view of cognition, based on the notion that functioning in the world with bodies fundamentally shapes cognitive processes (e.g., Niedenthal, Barsalou, Winkielman, Krauth-Gruber\& Ric, 2005; Smith \& Semin, 2004). As in other disciplines, the new focus on embodiment has generated enthusiasm and renewed interest in physical aspects of affective experience.

One of the main assumptions of embodied cognition ${ }^{2}$ is that ultimately, the goal of cognitive processes is not to produce mental representations of abstract knowledge, but instead, to facilitate appropriate action in the world. The kinds of actions that are possible, and therefore, the kinds of cognitive structures that follow, are constrained by stable as well as temporary characteristics of the human body. This view is not entirely new (e.g., Gibson, 1979; Merleau-Ponty, 1962); however, traditionally psychologists have tended to treat cognition as taking place entirely "in the head", and the role of the body has been largely underappreciated (for a history of the disembodied mind, see Johnson, 1987; Spellman \& Schnall, in press). To appreciate the excitement following this new perspective, it needs to be clarified what embodiment refers to. The term embodiment has been used by researchers in cognitive science in multiple ways, sometimes without being defined explicitly (Anderson, 2003; Wilson, 2002). Often it has been defined not so much by what it is, but by what it is not. For example, it has been noted that the goal of cognitive processes is not simply thinking. Researchers endorsing the embodied approach to cognition have put their perspective in opposition to the assumptions of more traditional approaches to cognition (e.g., Fodor, 1983; Fodor \& Pylyshyn, 1988). These research traditions, which have been termed objectivist (Lakoff \& Johnson, 1999) or cognitivist (Clark, 1997; Varela et al., 1991), make very specific claims

2 For purposes of simplification, the term "embodied cognition" is used to refer to a cluster of theoretical approaches that share, among other things, a commitment to the physicality of human cognition. It is acknowledged, as cautioned by Barsalou (2008), that the embodied component of such approaches is but one aspect, which downplays the fact that other aspects are necessary and equally important. As a more appropriate and inclusive term Barsalou (2008) suggests "grounded cognition." However, because the term "embodied cognition" has become popular, especially within social psychology, this term was chosen for discussion in the present paper. 
about the nature, and the purpose of internal representations. The issue is, to use an often quoted example, how human beings represent the fact that chairs have four legs, a seat, a back, possibly some armrests, and so on. Do people indeed represent objects, ideas, and situations in the form of features or propositions? Traditional theories assume that amodal symbols and properties define conceptual structure, and that cognitive processes correspond to the manipulation of those symbols (e.g., Fodor, 1983; Fodor \& Pylyshyn, 1988). The symbols themselves are arbitrary, and are given meaning only by means of how they are combined. This computational metaphor in cognitive science has been influential ever since the cognitive revolution.

In contrast to traditional theories of cognition, according to the embodied approach, the brain evolved not to provide an accurate mirror representation of the world, but rather, to help humans, and non-human animals alike, to successfully act in the world. Because the goal of mental representations is to allow for actions and interactions with the world, representations are the result of interactions with the environment, rather than consisting of arbitrary abstract symbols (Barsalou, 1999; Glenberg, 1997). Such embodied representations maintain the modality of perceptual experience, and concepts are considered to involve simulations of such perceptual processes (Barsalou, 1999).

This general claim is in part based on the notion of affordance, coined by Gibson (1979), which describes potential interactions between a person and an object, or an environment. Certain actions are "afforded" by certain objects, whereas others are not: A chair affords sitting on it, but normally does not afford walking on it. However, affordances are not fixed, but depend on specific circumstances. One can easily imagine situations where a chair will not afford sitting, for example, if it is a miniature model in a doll house. Similarly, one can imagine situations in which a chair will afford walking on it, for example when a series of chairs forms a walkable trail out of a flooded house. A critical implication of cognition in the service of action is that specific actions happen in specific contexts.

Importantly, context or situational factors do not simply modify what action, and thus, what cognitive process is appropriate, but rather, they define the action. Consider the example of the frog's visual system (Ingle, 1973). Frogs have several neurologically separate visual pathways, such as one pathway to detect prey, another to monitor predators, and yet another to control visually guided locomotion. Thus, frogs do not have a generalpurpose visual system that responds differently depending on what input it receives; rather, the input (e.g., prey vs. predator), and the corresponding action associated with the input (catching a fly vs. escaping from a hawk) determines the process of visual perception. The goal of vision is not to see, 
but to control movements as a response to stimuli in the environment (Milner \& Goodale, 1995). By rejecting the notion of "pure" vision (Marr, 1982), or more generally, the notion that perceptual processes are informationally encapsulated (Fodor, 1983) and independent of higher-level cognitive processes, embodied cognition approaches have provided a productive way of studying the interactions of vision and action (e.g., Tucker \& Ellis, 1998). One domain of perception for which the embodied view of cognitive processes has become especially relevant is the perception of the physical environment, including distances and slants of hills, as will be reviewed next.

\section{Embodied perception: Physiological resources}

Judging from their everyday experiences people assume that their visual perceptions accurately reflect the physical world around them. In contrast, inspired by Gibson's (1979) notion of affordance a view of perception based on the economy of action (Proffitt, 2006) proposes that people perceive the space around them relative to how they can act on it. For example, when exhausted due to a long and strenuous run people perceive hills as steeper than when they are not fatigued (Proffitt, Bhalla, Gossweiler, \& Midgett, 1995). Similarly, people who wear heavy backpacks perceive hills to be steeper than do people who are unencumbered (Bhalla \& Proffitt, 1999). Such studies have demonstrated an association between spatial perception and factors that are relevant to action: Because climbing a hill is challenging for fatigued or encumbered people, they perceive it be to steeper. Thus, people's perception of the physical world is not simply a function of objective features of the environment such as slant or extent, but is constrained by the perceiver's ability to act on that given space, at a given time. Evidence for the economy of action involved in making perceptual estimates has come from two sources: Studies involving hill slant, and studies involving distance. These will be reviewed in turn.

\subsection{Visual slant perception}

Various accounts of visual perception propose two distinct functions of vision that appear to be controlled by two anatomically separate visual pathways in the brain (e.g., Milner \& Goodale, 1995). The two visual processes relate to action planning and action execution, which are thought to be controlled by the ventral stream, and the dorsal stream of the visual cortex. Studies assessing the perception of hill slants have documented that people give 
different estimates depending on whether a slant measure taps into one function of vision, or the other (Bhalla \& Proffitt, 1999; Proffitt et al., 1995; Schnall et al., 2008): Action planning is captured by a verbal estimate that involves first, having the participant state the slant of a hill in geometric degrees, and second, by asking the participant to engage in visual matching, which involves adjusting a disk to represents the cross-section of the hill. The verbal and visual measures assess people's explicit awareness of slant, and on these measure people tend to dramatically overestimate hill slant. For example, research participants typically estimate a $5^{\circ}$ hill to be $20^{\circ}$, or a $10^{\circ}$ hill to be $30^{\circ}$. Such overestimation is amplified with experimental manipulations that would make it difficult for a person to climb a hill, such as being encumbered, or fatigued, as noted earlier.

In contrast, action execution is captured by a haptic measure of hill slant, which involves placing the dominant hand on a palmboard that can be adjusted, without sight of the hand, to be parallel to the hill's incline (see Figure 1). This visually-guided action measure is generally accurate and further, is not changed by manipulations of physiological state such as whether the person wears a heavy backpack or is fatigued due to previous exercise (Bhalla \& Proffitt, 1999). In other words, factors that would make it challenging to ascend a hill lead participants to estimate the hill as more steep on the verbal and visual measures, but do not change how steep they perceive it when using the haptic measure. How can this apparent disconnect

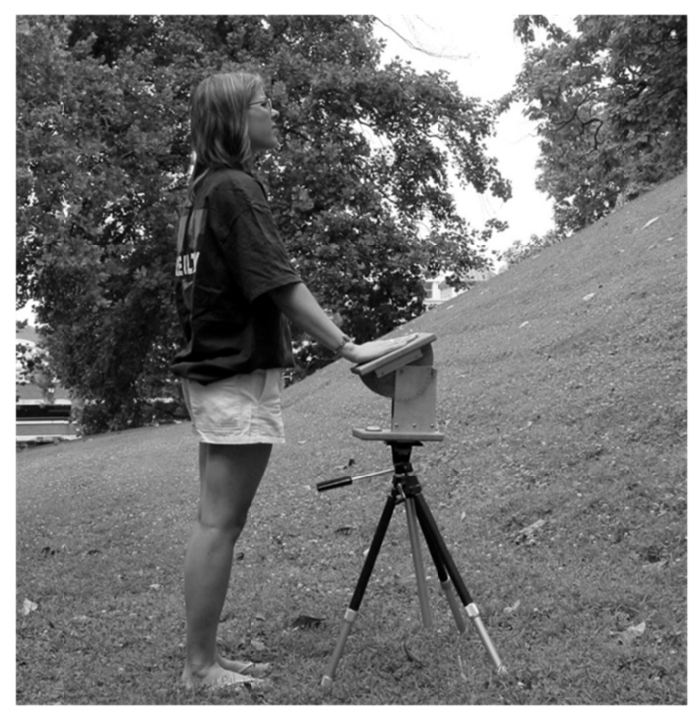

Figure 1. Participant using haptic measure to assess hill slant. 
between the two types of measures be explained? Importantly, the haptic palmboard measure is assessed by asking participants to place their hand on the palmboard without looking, and adjust it to be equivalent to the inclination of the hill in front of them. Therefore, there is no visual feedback when performing this task. In contrast, the explicit verbal and visual reports are made by explicitly deciding on the magnitude of hill slant. Thus, one way of thinking of this distinction is to consider the verbal and visual measures as accessible to explicit awareness because they are guided by deliberate manipulation of the measures, and allow the participant to adjust the measure while at the same time looking at the hill, whereas control of the haptic measure relies on visuomotor processes that are not entirely open to conscious consideration (see Witt \& Proffitt, 2007, for a detailed discussion).

The overestimation in the measures that relate to action planning is adaptive and is driven by two separate mechanisms (Proffitt, 2006). First, response compression allows for greater sensitivity to small changes in slant for relatively shallow slants, which are the only slants one can walk on and therefore the only slants for which small differences are of any relevance (see Proffitt, 2006, for further detail). Second, because these representations inform decisions about action that need to take into account costs and benefits, anticipating what would be involved in ascending the hill, what resources would be required, and what resources are available and including this information in explicit perception is adaptive in terms of influencing individuals to be cautious and "on the safe side" when planning future action and energy expenditure. In contrast, for actual navigation and movement within the environment the body needs to act precisely in line with the conditions of the environment: When ascending a steep hill a person needs to lift the foot such that it perfectly accommodates the ground upon which it will be placed and thus factors related to action planning are relatively irrelevant.

Using these convergent measures for which different results are expected has the benefit that effects due to experimental demand characteristics are unlikely, because in that case all measures should show the same pattern of results, which they do not.

\subsection{Visual distance perception}

Inspired by the initial finding that hills appear steeper when observers wear a heavy backpack relative to when they do not (Bhalla \& Proffitt, 1999), follow-up studies tested whether similar effects could be obtained when encumbered observers estimate distance to walkable targets ranging from 1 to 17 meters (Proffitt et al., 2003). In one study, participant stood in a grassy 
field and estimated how far from them orange traffic cones were that had been placed at varying distances. ${ }^{3}$ Indeed, participants wearing a heavy backpack estimated the distances to be farther than those who were not, which is consistent with the idea that on some level, perceivers took into account how effortful it would be to walk to specific targets given their current physical state. ${ }^{4}$ Other research had participants throw a heavy or a light ball at targets before estimating the distance to those targets. After throwing a heavy ball participants indicated that distance to the targets was farther than after throwing a light ball (Witt, Proffitt \& Epstein, 2004). Presumably, perceived effort when carrying out a specific action, in this case throwing a ball, was interpreted as being diagnostic of the distance to the object relating to that action.

Considerations of how easy or difficult it is to reach a target also influence estimates of distances to closer targets. Witt, Proffitt and Epstein (2005) presented participants with targets that were either just within reach of their arm or outside of arm's reach. Participants were seated in front of a table into which small white circles were projected. When the circle disappeared participants attempted to touch the location in which it had appeared, either using their finger, or using a baton. Targets were estimated as closer when reaching with the baton than when reaching with the finger. However, a follow-up experiment showed that reachability only influenced distance estimates when it was relevant to action, namely when the perceiver had previously formed an intention to reach, but not when merely holding the baton (Witt et al., 2005).

Of particular importance is that studies of embodied perception redefine what might be considered an "accurate", or "rational" response to the questions of "how far is it to get to a target location?" or "how steep is a hill?" On the one hand, if the hill is actually 5 degrees in incline, any deviation from 5 degrees is incorrect. However, on the other hand, when wearing a backpack the hill becomes functionally steeper, and an answer of 20 degrees might in fact be the appropriate, although factually "irrational" or "incorrect" answer. Overall, studies on embodied perception suggest that even seemingly objec-

3 In contrast to the studies on hill slant, these studies involving distance estimates only used "explicit" perceptual estimates in the form of participants giving estimates in feet and inches. No equivalent to the haptic measure was used.

4 In these studies, as in studies involving hill slant described above, experimental instructions to participants leave it ambiguous whether walking to the target (or climbing up the hill) would actually be required after making the perceptual estimates. However, underlying the notion of affordance is the assumption that people automatically perceive the environment with regard to specific actions, without needing an explicit goal to perform such actions. 
tive aspects of the physical environment, such as how far is it to get to a target, or how steep is an incline, are constrained by physical considerations of the body that perceives the environment.

\subsection{Direct evidence for the economy of action}

The economy of action account (Proffitt, 2006) maintains that spatial perceptions depend on the resources necessary to perform actions relative to the resources that a person has available. What kind of resources are required to deal with challenges in the environment? At the most basic level, human beings, like all other animals, are living systems in which energy is necessary to maintain bodily functions such as respiration, digestion, blood circulation and to support any physical activity that goes beyond the resting state. Whenever energy is used, it must be replaced. Carbohydrates supply energy in the form or glucose, which becomes available throughout the body via the bloodstream. Although any physical action requires energy, the precise amount depends on the properties of the environment in which the action takes place. For instance, walking up a $5^{\circ}$ hill requires much less energy than walking up a $20^{\circ}$ hill. Similarly, walking up a hill while carrying a heavy backpack requires more energy than when unencumbered. If the energetic requirements for an action are high or the available energy is low, then hills will appear steeper than they would otherwise, thereby discouraging an individual from performing energetically suboptimal actions.

The studies reviewed above involving visual slant and distance perception were built on the assumption of the economy of action. However, they did not experimentally manipulate or measure current energetic states of the perceiver. Furthermore, critics have objected that participants might have shown demand characteristics by indicating that the hill is steeper because they knew that they were wearing a backpack or were fatigued.

In recent work, Schnall, Zadra and Proffitt (in press) directly manipulated blood glucose levels, with the expectation that high blood glucose would make hills appear less steep relative to low blood glucose. Under the subterfuge of a taste testing study, participants were given a soft drink that, unbeknownst to them, was sweetened with either sugar, or with artificial sweetener. Thus, some participants were given readily available glucose, whereas the other participants were not. Then participants put on heavy backpacks while performing the same slant estimates as described above, including verbal, visual and haptic reports. Results from two experiments indicate that participants who had consumed a glucose-containing drink perceived the hill's slant to be less steep than did participants who had consumed a drink containing non-caloric sweetener. Because climbing a hill 
while wearing a heavy backpack poses a metabolic challenge, explicit action planning benefits from the apparent slant of the hill being influenced by one's potential to expend energy. In contrast, visual processes involved in action execution, as measured by the haptic estimates, were unaffected by energetic factors, because the biomechanics of climbing a hill remain unchanged. Schnall et al. (2009) thus argue that a process that is relatively effortless and automatic, namely visual perception, is influenced by glucose levels because visual perception serves as the "fuel gauge" of whether glucose is available or not, and what action in a given physical environment is possible.

\section{Embodied perception: Social and emotional factors}

In addition to the studies that investigated physiological resources, such as whether a person is rested or fatigued, has high or low levels of blood glucose available, and so on, several studies have started looking into the influence of more psychological processes such as emotion and motivation.

\subsection{Emotion and perception of space}

Riener, Stefanucci, Proffitt and Clore (2003) tested the influence of mood on hill slant perception. Mood was induced by having participants listen to happy music or sad music, or by having participants write about happy or sad life events. Then participants completed slant estimates of a steep hill. Sad participants judged the hill as being steeper than those in the happy condition. As was found previously, the visually guided action measure was unaffected across conditions.

Stefanucci and colleagues have explored the contribution of fear to various perceptual processes. Participants who stood at a skateboard (that was secured to be stationary) at the top of a hill and reported feeling afraid at the prospect of going down the hill perceived it to be steeper than participants who merely stood on a wooden box at the top of the hill (Stefanucci, Proffitt, Clore, \& Parekh, 2008). Going beyond perceptual estimates of slant and extent, Stefanucci further developed a new perceptual paradigm by assessing people's perception of height (Stefanucci \& Proffitt, 2009). Participants estimated distance either when looking down from a balcony, or when looking up to the balcony. Vertical distance involving the balcony was generally overestimated relative to equivalent horizontal distance. Furthermore, overestimation was particularly pronounced when looking down rather than when looking up, and when looking down from large height 
rather than small height, presumably because of the potential danger and fear associated with standing exposed on a high balcony. This finding is consistent with earlier work involving hill slant that had shown that hills are perceived as steeper when viewed from the top relative to being viewed from the bottom once their incline makes them too difficult to descend, although it might still be possible to ascend (Proffitt et al., 1995). Further, individuals with pronounced fear of heights overestimate vertical distance from a two-story balcony more than those who do not suffer from fear of heights, thus suggesting that perceptual bias can be symptomatic of underlying emotional processes (Teachman, Stefanucci, Clerkin, Cody, \& Proffitt, 2008). In addition to stable individual differences regarding fear of height, components of fear involving arousal have been manipulated experimentally. Stefanucci and Storbeck (2009) first exposed half of their participants to arousing visual images, while the other half of the participants were exposed to non-arousing visual images. Subsequent height estimates of a balcony were significantly higher for participants who previously had seen arousing stimuli, presumably because the arousal from the images intensified the fear associated with looking down from a high balcony. However, in contrast to such vertical distance estimates, high or low arousal did not have any influence on horizontal distance estimates, suggesting that arousal only has an impact when it is functionally relevant to the physical situation under consideration.

Further, testing motivational influences, Balcetis and Dunning (2007) demonstrated that manipulations of cognitive dissonance changed the perception of distance and slant. People in high choice conditions estimated slants to be less steep and distances to be less far than those in low choice or control conditions, presumably because they were keen to resolve dissonance.

Such findings involving emotional and motivational factors might be considered intuitive from a social psychologist's perspective; however, they are incompatible with most theories of perception that assume that perception constitutes a modular process that is independent from extraneous factors (Fodor, 1983; Marr, 1982). Instead, these findings are consistent with ecological approaches to perception (e.g., Gibson, 1979; Milner \& Goodale, 1995; Proffitt, 2006).

\subsection{Social resources and spatial perception}

Recent work has further explored whether other people might also be considered valuable resources in a manner that impacts spatial perception, and to some extent lead to effects parallel to the ones obtained on physiological 
resources. Indeed, the basic finding that social support is a beneficial resource on many levels is well established. Apparently the number and intensity of close social contacts serves as a buffer against life's adverse events (Thoits, 1986) and thereby reduces the risk of stress-related illnesses such as heart disease (Seeman \& Syme, 1987) and cancer (Fawzy et al., 1993). Stress responses to threatening situations are dampened when a person is in the presence of a supportive other compared to when being alone (Kamarck et al., 1990), and a similar attenuation of stress reactivity in the presence of conspecifics has been found even in non-human animals, including rats (Davitz \& Mason, 1955; Latané, 1969), guinea pigs (Hennessey, O'Leary, Hawke, \& Wilson, 2002) and monkeys (Gust, Gordon, Brodie, \& McClure, 1994). Overall, social support appears to "lighten the load" of individuals who find themselves in stressful and challenging situations.

If the presence of another person has such benefits, then the perception of challenging spatial environments such as steep hills might be modulated by the presence or absence of supportive others as well. Schnall, Stefanucci, Clore and Proffitt conducted a pilot study to test whether simply verbally invoking a friend's presence would have an effect on people's distance estimates. Participants were graduate students of the Psychology Department at the University of Virginia which is housed in Gilmer Hall. They were asked to complete a two-item survey which they received by email, estimating the distance from Gilmer Hall to the following two locations: The downtown mall, a popular destination approximately 2.3 miles from Gilmer Hall, and Monticello, Thomas Jefferson's former hill-top home, located about 5.8 miles from Gilmer Hall and involving an uphill journey. Half the participants were asked to imagine that they were standing in front of Gilmer Hall and needed to walk to the locations, and estimate how far it was to get there. The other half of the participants, however, was asked to imagine that "you and a friend are standing in front of Gilmer Hall", and needed to walk to the locations, and then give distance estimates. For the short, easily reachable location of the downtown mall mentioning the friend did not make any difference to distance estimate. For the more challenging, long distance to Monticello, however, participants who were asked to imagine walking with a friend found the distance to be significantly shorter than those who imagined walking alone. It seemed that when faced with a difficult situation, having to walk a long distance involving a steep hill, thinking of doing so while a friend was present made it seem less difficult. However, this was only preliminary evidence because it involved imagined or recalled distances, rather than actual spatial features of the environment that one is currently looking at.

Schnall, Harber, Stefanucci and Proffitt (2008) followed up on this suggestive work with more controlled studies involving slant estimates of a 
steep hill under conditions where social support was either present or absent. In the first study, participants stood at a steep hill while wearing a heavy backpack, and did so either alone, or with a friend standing at their side. Those who were with a friend, compared to those alone, perceived the hill as less steep. Thus, a psychosocial resource, social support, influenced apparent slant in much the same way as do energetic factors. Importantly, being with a friend versus being alone only affected measures related to explicit awareness and planning (verbal and visual estimates), and had no effect on the measure of visually guided action (haptic estimate). This pattern was consistent with earlier findings on energetic resources and slant estimates that implicate two different visual systems (Bhalla \& Proffitt, 1999; Proffitt et al., 1995). A second study manipulated social support by having participants first mentally image a positive, neutral, or negative social contact, and then estimate the slant of the hill. Indeed, participants who thought of a positive other estimated the hill to be less steep than participants who had either thought of a neutral or negative other. Again, differences between experimental conditions were only found on the verbal and visual measures, which are related to explicit awareness, but not on the haptic measure, which is related to the visual control of action. Critically, in both studies the quality of the supportive relationship mediated the effect on visual perception. In Study 1, friendship duration was negatively correlated with visual and verbal slant estimates: The longer a friend was known, the less steep the hill appeared. Further, in Study 2, the feelings of closeness to the imaged other were correlated to both the verbal and visual hill slop estimates: The closer participants felt toward their imaged social contacts, the less steep the hill appeared to them. Thus, the critical ingredients that make relationships a powerful psychosocial resource explained why relationships moderate perception.

The studies reviewed above illustrate how perceptions of space can change as a function of supportive others. However, whereas we like to be physically close to people who we feel emotionally "close" to, people prefer to literally maintain a distance to strangers or generally, people who they are not "close" to. More specifically, people maintain an area of personal space around them, and are very sensitive to violations of this personal space (Argyle \& Dean, 1965; Hall, 1968). Schnall, Witt, Stefanucci, Augustyn, Clore and Proffitt (2005) conducted a study modeled after field studies in naturalistic settings that involve an invasion of territory (e.g., Sommer, 1959). In contrast to those early studies that measured the person's behavioral response, we measured the person's distance estimate to an object after their personal space was violated. In the invasion condition, participants' space was invaded by an experimenter who casually placed a can of Coke from which she had been drinking immediately in front of 
them (at a distance of about $10-45 \mathrm{~cm}$ ). In the control condition, the experimenter retrieved a fresh can of Coke from her briefcase, and placed it in front of the participant, with the words, "This is for you for participating." Thus, the only difference between the two conditions was whether the experimenter had established ownership of the can (as indicated by drinking from it), and thus invaded the participant's personal space, or the participant had ownership of the can (as indicated by the experimenter's comment). Then participants gave a matching estimate of the distance between the Coke can and the edge of the table where they were sitting. Results showed that participants whose space had been invaded estimated the experimenter's can to be significantly closer than participants who had their own can within their personal space. Thus, when personal space was invaded, another person's object was experienced as "too close."

Several mechanisms for the observed effect are possible. First, the effect might be due to the fact that a social norm violation had taken place. Alternatively, it might be that an affective response was created, such as a feeling of disgust toward another person's beverage. Future studies are aimed at differentiating between these possibilities. What is clear, however, is that the perceived distance to an identical object can change dramatically depending on social constructs such as object ownership. Thus, people appear to not only use space in specific ways as a function of social relationships, but based on the results obtained in this study, also perceive space as a function of social relationships. It further appears that social processes are especially powerful in the action space within reach that has been termed personal space (Cutting \& Vishton, 1995) because this space coincides to some extent with the personal space that has been extensively studied by social psychologists (Argyle \& Dean, 1965; Hall, 1968).

All of these studies - whether about physical resources or psychosocial resources - are based on the assumption that perceptual processes depend on a person's resources in the context of navigating the environment. Thus, these studies were conducted from an embodied perspective, because the traditional cognitive model would not make different predictions for a person standing in front of an actual hill, versus sitting at a computer and indicating their response using keyboard presses.

\subsection{Subjective vs. objective judgments}

As noted earlier, a considerable body of research has demonstrated that subjective judgments of various kinds can be influenced by contextual factors (e.g., Clore et al., 2001). What makes perceptual judgments different, however, is the fact that they are concerned with objective judgments, 
namely with features of the physical environment such as distances, hill slant or height, for which there is an objectively correct answer. In contrast to judgments of life satisfaction, risk, moral contempt, etc., there is an objective standard that indicates whether the judgment was correct or not. However, although perceptual judgments can be compared to an external standard, as demonstrated in the research reviewed in this chapter, they are nevertheless influenced by a variety of contextual factors relating to both physiological and social resources. Although it might not be that surprising that a hill appears steeper to a person who is wearing a heavy backpack, such a finding is inconsistent with traditional cognitive theories that view visual perception as a low-level process and is only predicted by theories that consider embodied factors within perception. What might be even more surprising, however, is that emotional factors can have a similar effect: A hill appears steeper for a person who is afraid because of standing on a skateboard at the top of a hill. Might this mean that being afraid is functionally similar to wearing a heavy backpack? Or if consuming a sugary drink makes a hill look less steep, and being with a friend also makes a hill look less steep, does this mean that consuming energy in the form of sugar is functionally similar to being supported by a friend? At this point there is not sufficient empirical evidence to provide an answer of whether physiological and social resources might involve the same mechanism in terms of constraining perception of the physical space. However, all this work suggests that such factors are integral to the perceptual situation, and they need to be considered when trying to understand how people metaphorically and literally view the social and physical worlds.

\subsection{Spatial perception and spatial metaphors}

In their theory of conceptual metaphor, Lakoff and Johnson (1980; 1999) propose that concepts are represented in the form of metaphors that are grounded in basic experiences of how the body interacts with the physical world, for example, how people use resources, or how they move around in physical space. As a directly perceived, immediate concept, space can be used as a source of metaphors for various target domains. For instance, the spatial concept of verticality is used when describing positive or negative experiences, for example, when saying "We hit a peak last year, but it's been downhill ever since." (Lakoff \& Johnson, 1980, p. 17). Whereas early work on metaphor theory was conducted within linguistics and consisted of examples of metaphors and how they map onto physical experience, in recent years social psychological studies have confirmed such connections empirically (e.g., Jostmann, Lakens \& Schubert, 2009; Landau, Sullivan \& 
Greenberg, 2009; Meier \& Robinson, 2004; Schubert, 2005; Sherman \& Clore, 2009; Williams \& Bargh, 2008; Zhong, \& Leonardelli, 2008). For example, Meier and Robinson (2004) showed that positive words were categorized more quickly when presented in the top half of a computer screen relative to the bottom half of the screen, whereas the opposite was the case for negative words. Such results suggest that conceptual structure might indeed be represented in a form that makes reference to physical space, for example, an upright posture when feeling happy, versus a slumped, "depressed" posture when feeling sad.

The results reviewed above in the context of embodied perception raise an additional possbility of the mutual influence of metaphor and physical space: If being with a friend takes a "load of your shoulders" and makes a steep hill appear less steep, then perhaps the metaphoric representation of friendship and its effect on challenges in life feeds back into how such challenges are literally perceived. The notion of social "support" might indeed mean that somebody is there for you to help with a difficult "load", therefore decreasing the perceived slant of the hill. Similarly, when a target is "within reach" because a tool is available, it appears physically closer. Physical and metaphorical aspects of close vs. far experiences have recently been formalized within the framework of Construal Level Theory (see Liberman \& Förster, this volume). Future studies will need to determine the extent to which physical and metaphorical space interact with one another.

\section{Summary}

Traditionally visual perception has been conceptualized as a low-level process that takes place in a "computationally encapsulated" manner (e.g., Fodor, 1983). Such approaches have assumed that visual perception is a modular process that is invariant to "higher-level" processes such as the perceiver's goals or social context. However, two lines of recent research have challenged this assumption: First, studies involving maps and other conceptual representations of space, and second, studies involving the perception of extent and slant, or in other words, of distances and inclines. Studies on mental maps suggest that people do not represent physical space in a veridical manner, but instead, distort it to be in line with functional considerations, such as whether a map involves a border to another country, or some more abstract boundary. Further, based on Gibson's (1979) notion of affordances, studies on the perception of physical space suggest that people perceive the world around them as a function of how they would act in that world: When their current bodily resources make it difficult in principle to, for example, cover a distance, or climb a hill, such 
environments are perceived as challenging, with targets appearing far, and hills appearing steep. Although strictly speaking such perceptual processes do not accurately reflect the true geographic properties of space, in a functional way, they are adaptive, because they influence individuals' anticipated action. In other words, although a hill might objectively not be as steep as it looks when the observer wears a heavy backpack, the observer is nonetheless better off by either planning for a challenging ascent, or avoiding it altogether.

In addition to physiological considerations recent research suggest that social and emotional resources might constrain the perception of physical space as well. For example, positive moods can make a hill appear less steep relative to negative moods such as sadness and fear. Further, having a friend nearby can literally and metaphorically provide "support" when ascending a steep hill. Such findings are consistent with recent approaches on embodied cognition, which are based on the premise that cognitive processes follow from interactions of the person in the physical environment. As a consequence, distinctions of modular processes of cognition, perception and action become difficult to maintain; all these aspects of physical and psychological functioning are closely intertwined. Further, bodily metaphors might not only reflect perceptual experience of space, but might indeed feed back into those physical experiences themselves.

\section{References}

Alter, Adam L. \& Daniel M. Oppenheimer (2008). Effects of fluency on psychological distance and mental construal (or why New York is a large city, but New York is a civilized jungle). Psychological Science, 19, 161-167.

Anderson, Michael L. (2003). Embodied cognition: A field guide. Artificial Intelligence, 149, 91-130.

Argyle, Michael \& Janet Dean (1965). Eye-contact, distance and affiliation. Sociometry, 28, 289-304.

Barsalou, Lawrence W. (1999). Perceptual symbol systems. Behavioral and Brain Sciences, 22, 577-660.

Barsalou, Lawrence W. (2008). Grounded cognition. Annual Review of Psychology, 59, 617-645.

Bhalla, Mukul \& Dennis R. Proffitt (1999). Visual-motor recalibration in geographical slant perception. Journal of Experimental Psychology: Human Perception and Performance, 25, 1076-1096.

Balcetis, Emily \& David Dunning (2007). Cognitive dissonance and the perception of natural environments. Psychological Science, 18, 917-921.

Bugmann, Davi \& Simone Schnall (2009). Negative affect and distance estimates on maps. Manuscript under review. 
Burris, Christopher T. \& Nyla R. Branscombe (2005). Distorted distance estimation induced by a self-relevant national boundary. Journal of Experimental Social Psychology, 41, 305-312.

Carbon, Claus-Christian \& Helmut Leder (2005). The wall inside the brain: Overestimation of distances crossing the former Iron Curtain. Psychonomic Bulletin and Review, 12, 746-750.

Clark, Andy (1997). Being there: Putting the brain, body, and world together again. Cambridge, MA: MIT Press.

Clore, Gerald L., Robert S. Wyer, Bruce Dienes, Karen Gasper, Carol Gohm \& Linda Isbell (2001). Affective feelings as feedback: Some cognitive consequences. In Leonard L. Martin \& Gerald L. Clore (Eds.), Theories of mood and cognition: A user's handbook (pp. 27-62). Mahwah, NJ: Erlbaum.

Coren, Stanley \& Joan S. Girgus (1980). Principles of perceptual organization and spatial distortion: The Gestalt illusions. Journal of Experimental Psychology: Human Perception and Performance, 6, 404-412.

Cutting, James E. \& Peter M. Vishton (1995). Perceiving layout: The integration, relative dominance, and contextual use of different information about depth. In William Epstein \& Sheena Rogers (Eds.), Handbook of Perception and Cognition: Vol. 5: Perception of Space and Motion. NY: Academic Press.

Davitz, Joel R. \& Donald J. Mason (1955). Socially facilitated reduction of a fear response in rats. Journal of Comparative and Physiological Psychology, 48, $149-156$.

Fodor, Jerry A. (1983). The modularity of mind. Cambridge, MA: MIT Press.

Fodor, Jerry A. \& Zenon W. Pylyshyn (1988). Connectionism and cognitive architecture: A critical analysis. Cognition, 28, 3-71.

Gibson, James J. (1979). The ecological approach to visual perception. Boston, MA: Houghton Mifflin.

Glenberg, Arthur M. (1997). What memory is for. Behavioral and Brain Sciences, $20,1-55$.

Golledge, Reginald G. (1987). Environmental cognition. In Daniel Stokols, Irwin Altman (Eds). Handbook of Environmental Psychology (131-174). John Wiley \& Sons.

Gust, Deborah A., Thomas P. Gordon, Anne R. Brodie \& Harold M. McClure (1994). Effect of preferred companion in modulating stress in adult female rhesus monkeys. Physiology and Behavior, 55, 681-684.

Haidt, Jonathan, Clark McCauley \& Paul Rozin (1994). Individual differences in sensitivity to disgust: A scale sampling seven domains of disgust elicitors. Personality and Individual Differences, 16, 701-713.

Hall, Edward T. (1968). Proxemics. Current Anthropology, 9, 83-95.

Hayduk, Leslie A. (1983). Personal space: Where we now stand. Psychological Bulletin, 94, 293-335.

Hennessey, Michael B., Shonagh K. O’Leary, Jesse L. Hawke \& Shannon E. Wilson (2002). Social influences on cortisol and behavioral responses of preweaning, periadolescent, and adult guinea pigs. Physiology and Behavior, 76, 305-314.

Ingle, David (1973). Two visual systems in the frog, Science, 181, 1053-1055. 
Johnson, Mark (1987). The body in the mind: The bodily basis of meaning, imagination, and reason. Chicago: University of Chicago Press.

Jostmann, Nils B., Daniël Lakens \& Thomas W. Schubert (2009). Weight as an embodiment of importance. Psychological Science, 20, 1169-1174.

Kamarck, Thomas W., Stephen B. Manuck \& J. Richard Jennings (1990). Social support reduces cardiovascular reactivity to psychological challenge: A laboratory model. Psychosomatic Medicine, 52, 42-58.

Kerkman, Dennis D., David Stea, Karen Norris \& Jennifer L. Rice (2004). Social attitudes predict biases in geographic knowledge. The Professional Geographer, 56, 258-269.

Kleck, Robert E. (1968). Effects of stigmatizing conditions on the use of personal space. Psychological Reports, 32, 111-118.

Lakoff, George \& Mark Johnson (1980). Metaphors we live by. Chicago: University of Chicago Press.

Lakoff, George \& Mark Johnson (1999). Philosophy in the flesh: The embodied mind and its challenge to Western thought. New York: Basic Books.

Landau, Mark J., Daniel Sullivan \& Jeff Greenberg (2009). Evidence that selfrelevant motivations and metaphoric framing interact to influence political and social issues. Psychological Science, 20, 1421-1427.

Latané, Bibb (1969). Gregariousness and fear in laboratory rats. Journal of Experimental Social Psychology, 5, 61-69.

LeBoef, Robyn A. \& Eldar Shafir (2006). The long and short of it: Physical anchoring effects. Journal of Behavioral Decision Making, 19, 393-406

Liberman, Nira \& Jens Förster (this volume). Estimates of spatial distance: A Construal Level Theory perspective.

Little, Kenneth B. (1965). Personal space. Journal of Experimental Social Psychology, 1, 237-247.

Marr, David (1982). Vision. San Francisco: Freeman.

Meier, Brian P. \& Michael D. Robinson (2004). Why the sunny side is up: Associations between affect and vertical position. Psychological Science, 15, 243-247.

Merleau-Ponty, Maurice (1962). Phenomenology of perception. London: Routledge.

Milner, A. David \& Melvyn A. Goodale (1995). The visual brain in action. Oxford: Oxford University Press.

Niedenthal, Paula M., Lawrence W. Barsalou, Piotr Winkielman, Sylvia KrautGruber \& François Ric (2005). Embodiment in attitudes, social perception, and emotion. Personality and Social Psychology Review, 9, 184-211.

Patterson, Miles L. (1977). Interpersonal distance, affect, and equilibrium theory. Journal of Social Psychology, 101, 205-214.

Proffitt, Dennis R., Mukul Bhalla, Rich Gossweiler \& Jonathan Midgett (1995). Perceiving geographical slant. Psychonomic Bulletin and Review, 2, 409-428.

Proffitt, Dennis R. (2006). Embodied perception and the economy of action. Perspectives on Psychological Science, 1, 110-122.

Proffitt, Dennis R., Jeanine Stefanucci, Tom Banton \& William Epstein (2003). The role of effort in perceived distance. Psychological Science, 14, 106-112. 
Riener, Cedar R., Jeanine K. Stefanucci, Dennis Proffitt \& Gerald L. Clore (2003). An effect of mood on perceiving spatial layout. Journal of Vision, 3, 227.

Schnall, Simone, Jennifer Benton \& Sophie Harvey (2008). With a clean conscience: Cleanliness reduces the severity of moral judgments. Psychological Science, 19, 1219-1222.

Schnall, Simone, Jonathan Haidt, Gerald L. Clore \& Alexander H. Jordan (2008). Disgust as embodied moral judgment. Personality and Social Psychology Bulletin, 34, 1096-1109.

Schnall, Simone, Kent D. Harber, Jeanine Stefanucci \& Dennis R. Proffitt (2008). Social support and the perception of geographical slant. Journal of Experimental Social Psychology, 44, 1246-1255.

Schnall, Simone, Jessica K. Witt, Jason Augustyn, Jeanine Stefanucci, Dennis R. Proffitt \& Gerald L. Clore (2005). Invasion of personal space influences perception of spatial layout. Journal of Vision, 5, 198.

Schnall, Simone, Jonathan R. Zadra \& Dennis R. Proffitt (in press). Direct evidence for the economy of action: Glucose and the perception of geographical slant. Perception.

Schubert, Thomas W. (2005). Your Highness: Vertical positions as perceptual symbols of power. Journal of Personality and Social Psychology, 89, 1-21.

Schwarz, Norbert \& Gerald L. Clore (1983). Mood, misattribution, and judgments of well-being: Informative and directive functions of affective states. Journal of Personality and Social Psychology, 45, 513-523.

Seeman, Teresa E. \& S. Leonard Syme (1987). Social networks and coronary artery disease: A comparison of the structure and function of social relations as predictors of disease. Psychosomatic Medicine, 49, 341-354.

Sherman, Gary D. \& Gerald L. Clore (2009). The color of sin: White and black are perceptual symbols of moral purity and pollution. Psychological Science, 20, 1019-1025

Smith, Eliot R. \& Gün R. Semin (2004). Socially situated cognition: Cognition in its social context. In M.P. Zanna (Ed.), Advances in Experimental Social Psychology: Academic Press.

Sommer, Robert (1959). Studies in personal space. Sociometry, 22, 247-260.

Spellman, Barbara A. \& Simone Schnall (in press). Embodied rationality. Queens' Law Journal.

Stefanucci, Jeanine K. \& Dennis R. Proffitt (2009). The roles of altitude and fear in the perception of height. Journal of Experimental Psychology: Human Perception and Performance, 35, 424-438.

Stefanucci, Jeanine K., Dennis R. Proffitt, Gerald L. Clore \& Nazish Parekh (2008). Skating down a steeper slope: Fear influences the perception of geographical slant. Perception, 37, 321-323.

Stefanucci, Jeanine K. \& Justin Storbeck (2009). Don't look down: Emotional arousal elevates height perception. Journal of Experimental Psychology: General, 138, 131-145. 
Teachman, Bethany A., Jeanine K. Stefanucci, Elise M. Clerkin, Meghan W. Cody \& Dennis R. Proffitt (2008). A new mode of fear expression: Perceptual bias in height fear. Emotion, 8, 296-301.

Thoits, Peggy A. (1986). Social support as coping assistance. Journal of Consulting and Clinical Psychology, 54, 416-423.

Thorndyke, Perry W. (1981). Distance estimation from cognitive maps. Cognitive Psychology, 13, 526-550.

Tucker, Mike \& Rob Ellis (1998). On the relations of seen objects and components of potential actions. Journal of Experimental Psychology: Human Perception and Performance, 24, 830-846.

Tversky, Amos \& Daniel Kahneman (1981). The framing of decisions and the psychology of choice. Science, 211, 453-458.

Tversky, Barbara (2000). Levels and structure of cognitive mapping. In R. Kitchin \& S.M. Freundschuh (Eds.). Cognitive mapping: Past, present and future. London: Routledge.

Varela, Francisco J., Evan T. Thompson \& Eleanor Rosch (1991). The embodied mind: Cognitive science and human experience. Cambridge, MA: MIT Press.

Wilson, Margaret (2002). Six views of embodied cognition. Psychonomic Bulletin and Review, 9, 625-636.

Williams, Lawrence E. \& John A. Bargh (2008). Experiencing physical warmth promotes interpersonal warmth. Science, 322, 606-607.

Willis, Frank N. (1966). Initial speaking distance as a function of the speaker's relationship. Psychonomic Science, 5, 221-222.

Winkielman, Piotr, Norbert Schwarz, Tedra A. Fazendeiro \& Rolf Reber (2003). The hedonic marking of processing fluency: Implications for evaluative judgment. In Jochen Musch \& Karl Christoph Klauer (Eds.), The psychology of evaluation: Affective processes in cognition and emotion (pp. 189-217). Mahwah, NJ: Erlbaum.

Witt, Jessica K., Dennis R. Proffitt \& William Epstein (2004). Perceiving distance: A role of effort and intent. Perception, 33, 577-590.

Witt, Jessica K., Dennis R. Proffitt \& William Epstein (2005). Tool use affects perceived distance but only when you intend to use it. Journal of Experimental Psychology: Human Perception and Performance, 31, 880-888.

Witt, Jessica K. \& Dennis R. Proffitt (2007). Perceived slant: A dissociation between perception and action. Perception, 36, 249-257.

Zajonc, Robert B. (1968). Attitudinal effects of mere exposure. Journal of Personality and Social Psychology, 9(2, Pt. 2), 1-27.

Zhong, Chen-Bo \& Geoffrey J. Leonardelli (2008). Cold and lonely: Does social exclusion literally feel cold? Psychological Science, 19, 838-842. 